\title{
Complete and absolute photonic bandgaps in highly symmetric photonic quasicrystals embedded in low refractive index materials
}

\author{
M.E. Zoorob ${ }^{\mathrm{a}, *}$, M.D.B. Charlton ${ }^{\mathrm{a}}$, G.J. Parker ${ }^{\mathrm{a}}$, J.J. Baumberg ${ }^{\mathrm{a}, \mathrm{b}}$, M.C. Netti ${ }^{\mathrm{b}}$ \\ ${ }^{a}$ Department of Electronics and Computer Science, University of Southampton, Southampton S017 1BJ, UK \\ ${ }^{\mathrm{b}}$ Department of Physics and Astronomy, University of Southampton, Southampton SO17 1BJ, UK
}

\begin{abstract}
It is firmly established that periodic lattice structures can support photonic bandgaps (PBG). However, complete and absolute photonic bandgaps (CAPBG) have only been achieved in high dielectric constant mediums such as GaAs ( $\varepsilon=13.6)$. An artificial quasiperiodic photonic crystal based on the random square-triangle tiling system was designed and fabricated. The photonic quasicrystal possesses 12-fold symmetry and was analysed using a finite difference time domain (FDTD) approach. High orders of symmetry in photonic quasicrystals have been shown to provide isotropic bandgaps across all the directions of propagation of light. As an outcome of these properties, this new class of photonic quasicrystal has been shown, for the first time, to possess a secondary non-directional CAPBG for a relatively low index material, silicon nitride $(\varepsilon=4.08)$. These materials are compatible with integrated optical technologies. This allows the fabrication of efficient integrated optical PBG devices such as WDM filters and multiplexers to become a real possibility. (C) 2000 Elsevier Science S.A. All rights reserved.
\end{abstract}

Keywords: Photonic quasicrystals; Photonic bandgaps; Finite difference time domain method; Symmetry

\section{Introduction}

Photonic crystals (PCs) have gained much attention in the past decade due to the pioneering work of $\mathrm{E}$. Yablonovitch [1]. It was noticed that artificial photonic crystals have the unique ability of inhibiting the propagation of electromagnetic waves over certain energy bands. These energy stop bands were termed photonic bandgaps (PBG). This sparked the possible use of such photonic crystals for the inhibition of spontaneous emission in active electro-optic devices [1]. Subsequently, many more equally important applications have been devised for PBG structures, these include passive devices such as wavelength division multiplexers, distributed feedback mirrors, high-Q cavities [2], optical waveguides, $90^{\circ}$ waveguide bends and waveguide splitters [3].

The most popular method for the formation of a photonic crystal involves the etching of a regular twodimensional lattice of air rods in a dielectric medium.

\footnotetext{
* Corresponding author. Tel.: + 44-1703-592493; fax: + 44-2380593029.
}

This regular structure creates a strong periodic modulation in the dielectric constant of the material, giving rise to a PBG. For many applications involving the use of PCs it is desirable to acquire complete and absolute photonic bandgaps [4]. In the case of such PBG structures, the wave propagation is forbidden for any direction of propagation, and independent of the polarisation state. This would provide the ideal PBG structure for the use in applications where the inhibition of spontaneous emission is desirable. It is firmly established that periodic triangular and hexagonal lattice structures can support complete photonic bandgaps in 2D and 3D [4-6]. However, complete and absolute PBGs (CAPBGs) have only been achieved in very high dielectric constant materials like GaAs $(\varepsilon=13.6)$ using hexagonal lattice structures [6]. The frequency range of the bandgaps formed by such a structure is also highly dependent upon the direction of wave propagation. Furthermore, large air pore diameters (large air-filling fractions) are required to create even small CAPBGs. Our experimental investigations [3] have shown that increasing the air-filling fraction can increase the losses of PCs due to the escape of light from the top of the surface. 
To alleviate such problems, the search for isotropic bandgaps (that is direction independent) in materials with low dielectric constants and small air filling fractions was undertaken. The use of low index materials facilitates the creation of novel optical devices which are highly compatible with optical fibre network systems. Such devices could include polarisation insensitive, direction independent WDM filters and multiplexers. These criteria prompted an investigation into high symmetry order photonic quasicrystals. In this paper we describe a new class of structure which is shown to possess a non-directional secondary photonic bandgap at visible wavelengths, for a relatively low index material (silicon nitride $\varepsilon=4.08$ ) and a relatively low air filling fraction $(\beta=0.28)$. Seconary bandgaps could be used to inhibit high-order radiative transitions in active opto-electronic devices. For the purpose of ease of investigation, experiments were performed at visible wavelengths. Furthermore, secondary PBGs reside at short wavelengths, aliminating the need of small lattice pitch PCs.

Based on previous theoretical studies $[4,5,7,8]$ it is evident that the anisotropy of the PBG is dependent on the symmetry of the photonic crystal lattice. This can be readily verified when band diagrams for PCs possessing a square lattice (four-fold symmetry) and a triangular lattice (six-fold symmetry) are compared. It is noticed that the higher symmetry triangular lattice $\mathrm{PC}$ has a more isotropic PBG as opposed to the square lattice structure.

As the order of symmetry in the photonic crystal increases, the Brillouin zone (BZ) becomes more circular. The highest level of symmetry found in natural crystals is six, however much higher orders of symmetry can be achieved in quasicrystals since these are fabricated artificially. High orders of symmetry have a tendency to increase the likelihood of the formation of degenerate states [7]. This in turn may reduce the overall PBG width due to available energy states coin- ciding within the PBG. However, we have found that due to the highly isotropic structure of the PBG, larger CAPBGs can be achieved even though the overall PBG width for specific wave propagation directions maybe reduced by the degenerate states.

A photonic quasicrystal based on the random squaretriangle tiling system possessing 12 -fold symmetry was analysed using a two-dimensional finite difference timedomain method (FDTD) [9]. This structure was designed to be compatible with integration in a silicon nitride waveguide and was intended for optical characterisation in the visible/near infrared region of the electromagnetic spectrum, where a wide range of laser sources and detectors are available.

\section{Implementation of tiling scheme in the quasicrystal structure}

The photonic quasicrystal structure is generated by using a random ensemble of squares and equilateral triangles. The random-Stampfli inflation rule [10] is applied to the ensemble of squares and triangles to generate the highly symmetric nature of the quasicrystal. In order for the structure to exhibit long-range 12-fold symmetry, several recursions of the randomStampfli inflation rule are required. A complete reference to the random-Stampfli inflation method for the generation of a maximally random square-triangle tiling is presented in M. Oxborrow et al. [10]. The dashed lines in Fig. 1 (a) demonstrate the parent dodecagon unit cell used for the inflation scheme. The solid lines show the tiling of the offspring dodecagons in the dilated parent cell. The ensemble of squares and triangles residing in any parent dodecagon can take up two unique orientations. This can be achieved by the application of a $30^{\circ}$ rotation on the parent dodecagon. Hence, a degree of randomness is incurred on the structure by the selection between normal and rotated

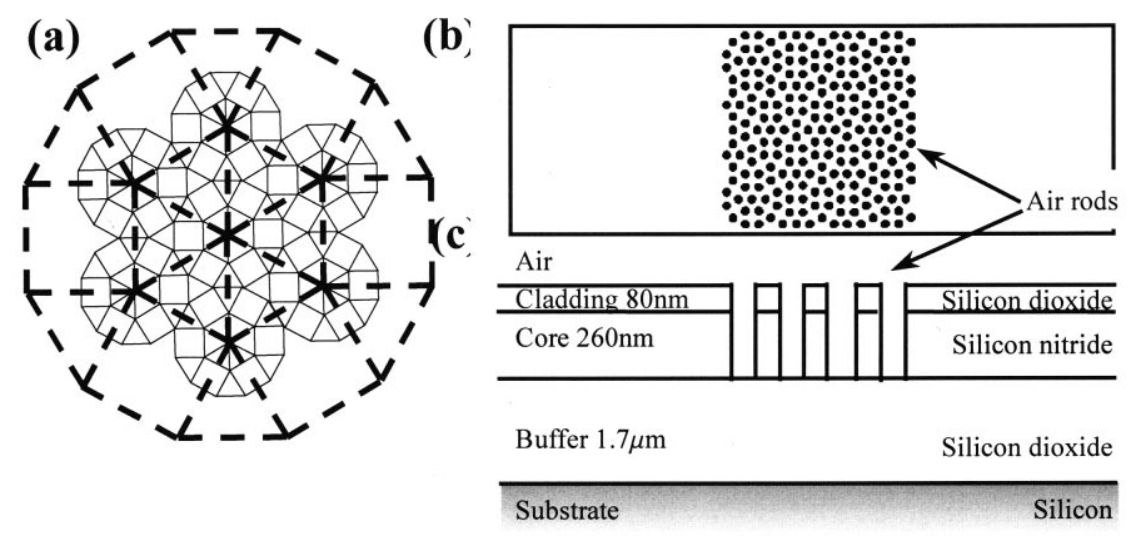

Fig. 1. (a) Random-Stampfli inflation of square-triangle tiling. Dashed line represents the parent tiling. Solid line represents the offspring tiling. (b) Top view highlighting the air rods arrangement. (c) Cross sectional view of the waveguiding structure (not to scale). 

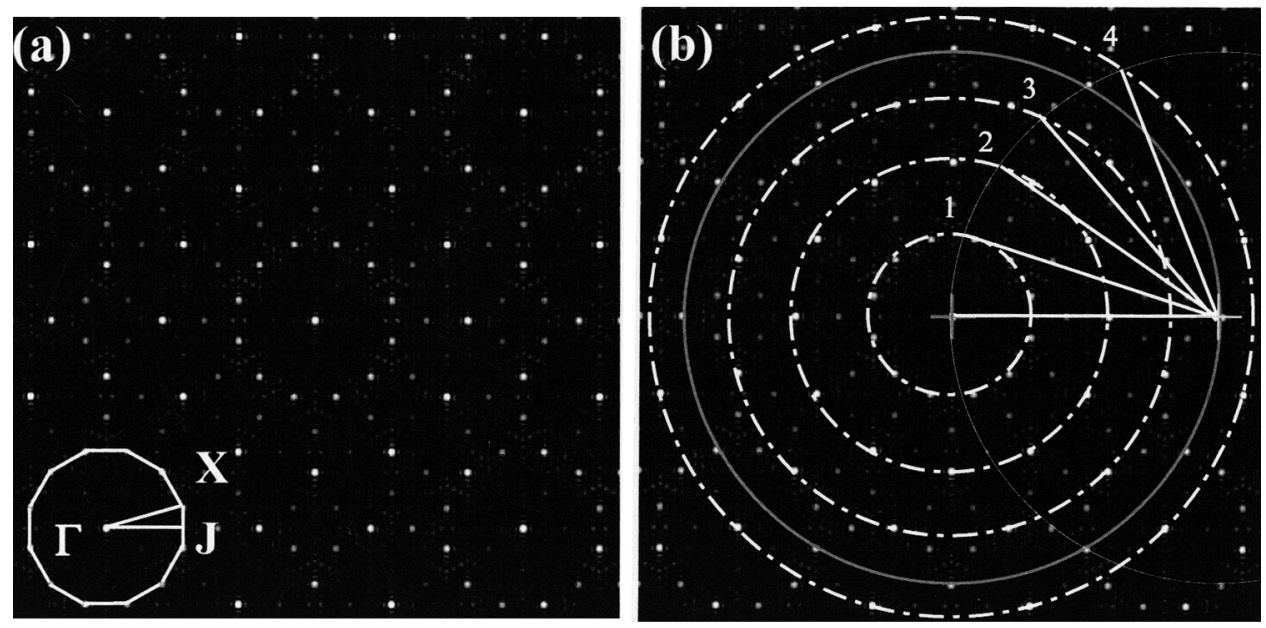

Fig. 2. (a) Diffraction pattern of the photonic quasicrystal with its accompanying Brillouin zone. (b) Ewald construction scheme. Dashed-dotted circles represent the projection of bright Bragg peaks. The white solid lines indicate the intersection of the Ewald sphere with the different Bragg peaks. These correspond to diffracted beams.

parent dodecagon unit cells before the application of the inflation rule. During the inflation scheme, the parent dodecagon is reduced in size by a ratio of $(\sqrt{3})+2$ to give rise to the offspring dodecagons. The centres of the offspring dodecagons are superimposed onto the vertices of every square or triangle in the parent unit cell. The air pores of the photonic quasicrystal are located at the vertices of every square and triangle. The distance between neighbouring air pores is defined by the pitch $a$. Fig. 1 (b) shows the resulting arrangement of the air rods in the dielectric material to be investigated, alongside Fig. 1 (c) which shows the cross section of the waveguiding structure.

The photonic quasicrystals examined are composed of $150 \mathrm{~nm}$ diameter air rods arranged on a pitch $a$ of $260 \mathrm{~nm}$, surrounded by a $260 \mathrm{~nm}$ sandwich of silicon nitride in silicon dioxide to confine the light in the 2D plane (Fig. 1 (c)).

\section{Modelling}

The diffractive properties of the quasicrystal were initially examined by the investigation of the quasicrystal in reciprocal lattice space (Fig. 2 (a)). The large parent cell generates the dodecahedral $\mathrm{BZ}$, accompanied by the irreducible triangular $\mathrm{BZ}$ indicated by the solid white lines. The real lattice vector $a$ extends out to the edges of Fig. 2 (a), defining the basic reciprocal lattice vector $2 \pi / a$. The 12 central bright Bragg spots in Fig. 2 (a) confirm the 12-fold symmetry of the photonic quasicrystal. Fig. 2 (b) illustrates the Ewald sphere construction. The central Bragg peak is selected as the reference point for the construction scheme. The dashed-dotted circles are construction lines. These indicate the equi-distant paths joining any bright Bragg peak and the central reference point. Construction lines for the weaker Bragg peaks are not shown for improved clarity. The lines represent the reciprocal lattice vector $\boldsymbol{k}_{\mathrm{G}}$. The solid grey arc represents the Ewald sphere segment for the wavelength $\lambda=633 \mathrm{~nm}$, with the reciprocal vector $\boldsymbol{k}_{\text {in }}=2 \pi n_{\text {eff }} / \lambda$. It is noted that an effective refractive index $n_{\text {eff }}=1.98$ is taken into account rather than the absolute refractive index $n$ of the silicon nitride material for better matching with experimental results. Identical $n_{\text {eff }}$ are assumed for all $\boldsymbol{k}$ vectors. This approximation can be justified due to the highly isotropic BZ. The intersection of the grey circle with the construction lines determines the projection angle of the far-field diffraction pattern at $633 \mathrm{~nm}, \boldsymbol{k}_{\mathrm{diff}}=\boldsymbol{k}_{\mathrm{in}}+\boldsymbol{k}_{\mathrm{G}}$ These are indicated by the solid white lines. In theory, this quasicrystal would be expected to diffract incident light into 12 beams. However, in practice, diffracted light from weak intensity Bragg peaks are not visible.

Transmission plots for all wave propagation directions were calculated using the two-dimensional FDTD method. The FDTD method was based on a centred difference spatial step scheme [10] and an exponentially decaying absorbing boundary condition was applied. A discretised form of Maxwell's equations was used to describe the electromagnetic field inside the dielectric medium. A centred difference spatial step was implemented to minimise transport errors of scattered waves inside the structure. The PBG structure was discretised using a grid of $1000 \times 1000$ nodes across 30 rows of the structure. The spatial increment $(\Delta h)$ was set to $\lambda / 50$ with respect to the minimum wavelength considered $\lambda=350 \mathrm{~nm}$. The time increment $(\Delta t)$ was fixed at $\Delta h / 50 c$ with respect to $\Delta h$, where $c$ is the speed of light in a vacuum.

The initial input fields were composed of Gaussian wavepackets in the spatial domain. Sampling points at 
the output of the photonic quasicrystal collect the field profile of the required electric or magnetic field component as a function of the sampled time. A fast Fourier transform (FFT) algorithm is applied to the collected data to provide the transmission characteristics for the photonic quasicrystal. The transmission plots are normalised to a control simulation for propagating Gaussian wavepackets in the dielectric medium. At each sampling node 14000 data points are collected to provide enough resolution for the FFT.

For the calculation of the transmission at angles not normal to the plane of incidence, the photonic quasicrystal is rotated about its centre to the desired angle and subsequently the finite differencing mesh is applied to the structure.

Although the main purpose of the investigation was the study of the secondary PBG, it is useful to make comparisons with features of the primary PBG. Fig. 3 shows the theoretically predicted primary bandgap size as a function of the air-filling fraction $(\beta)$ for normal incidence $(\Gamma \mathrm{J})$ to the photonic quasicrystal. The photonic quasicrystals modelled had a constant pitch $a=$ $260 \mathrm{~nm}$, identical to that described in Fig. 1 (c). The dielectric constant for silicon nitride core medium was assumed to be $\varepsilon_{\mathrm{b}}=4.08$. It is noticed that even for very small $\beta$ values a bandgap opens up in the photonic quasicrystal. At $\beta=10 \%$ a gap-midgap ratio of $12.9 \%$

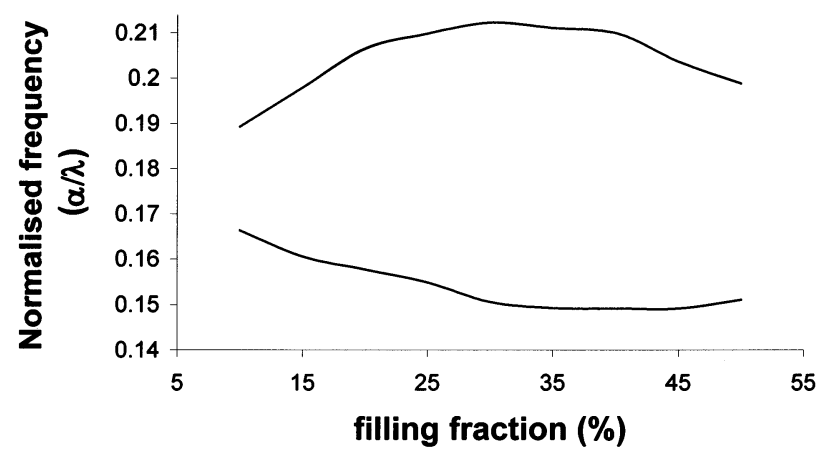

Fig. 3. Photonic bandgaps for normal incidence to the photonic quasicrystal versus different air-filling fractions.

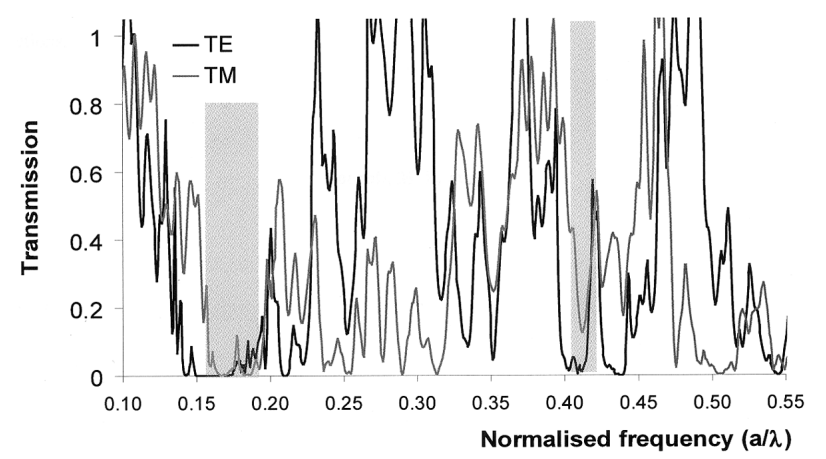

Fig. 4. Superimposed TE and TM transmission plots for the photonic quasicrystal in the $\Gamma \mathrm{J}$ propagation directions. is calculated for the $\Gamma \mathrm{J}$ propagation direction, while for a simple triangular lattice with the same filling fraction and the same dielectric material, a gap-midgap ratio of $2.5 \%$ is calculated for the $\Gamma \mathrm{J}$ propagation direction. Even though the simulations are only for $\Gamma J$ directions, due to the high isotropy of the PBG the bandgap remains open for all wave propagation directions, contrary to the triangular photonic crystal structures.

Further simulations were undertaken to highlight the degree of isotropy of the PBG. A photonic quasicrystal having a pitch spacing of $a=260 \mathrm{~nm}$ and an air filling fraction of $\beta=28 \%$ was investigated. Transmission spectra were calculated for several different directions of propagation between $\Gamma J$ and $\Gamma X$. using the FDTD method. Fig. 4 shows the predicted transmission for both TE and TM polarised modes along the $\Gamma \mathrm{J}$ direction. Maximum variations of $4 \%$ were observed between different wave propagation directions. For clarity only the direction with the most extreme PBG variation is shown. The overall width of the complete PBG is in-between 0.158 and $0.190 a / \lambda$. This gives rise to a $65.5 \%$ overlap in the complete PBG for the two different polarisation states as opposed to no CAPBGS for triangular and hexagonal lattice [6] structures for the same air filling fraction. This can be directly compared with the second complete PGB residing between 0.405 and $0.420 a / \lambda$. The gap-midgap ratio $\left(\Delta \omega / \omega_{0}\right)$ for the TE mode is $5.9 \%$, while that of the TM modes is $3.7 \%$. In both cases, it is evident that the TM bandgap lies in the middle of the TE bandgap domain, giving rise to CAPBGs. In the long wavelength limit (normalised frequencies, $\omega a / 2 \pi c$, less than $0.10 a / \lambda$ ) the transmission extends out to unity. At these wavelengths the fine structure of the square-triangle tiling is not resolved by the propagating waves, and the quasicrystal is seen as a homogeneous material of reduced refractive index.

The bandgaps of photonic quasicrystals were found to occur at lower frequencies compared to triangular and hexagonal photonic crystals. The normalised frequencies at the centre of the primary photonic bandgap $\left(\omega_{0}\right)$ for TE and TM polarised light are 0.170 and 0.176 $a / \lambda$, respectively. In contrast to regular lattice structures, nearest neighbour lattice vectors no longer define the BZ. As shown in Fig. 2 (a) the BZ in reciprocal space is defined by the dashed parent dodecagon in Fig. 1 (a) which joins lattice vectors several periods aways.

Time averaged FDTD methods were used to investigate the TE and TM mode profiles inside the photonic quasicrystal. Fig. 5 demonstrates the confinement of a TE mode in the photonic quasicrystal. The TE mode is incident from the right hand side of the structure at a wavelength of $\lambda=1100 \mathrm{~nm}$. This $\lambda$ residing within the primary bandgap of the structure. The localisation of light in the high dielectric regions is apparent. 


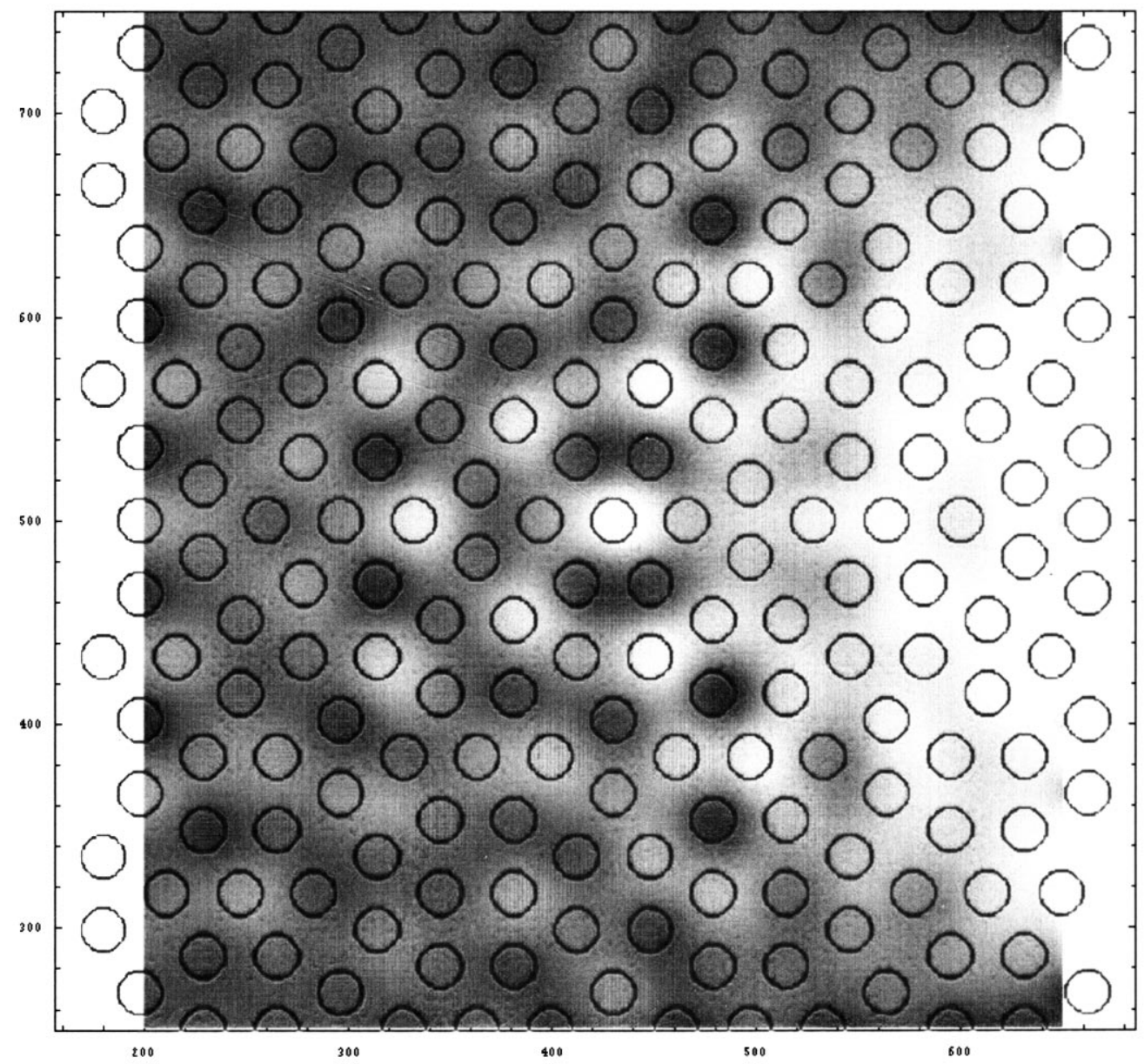

Fig. 5. Time averaged TE mode profile in photonic quasicrystal for $\lambda=1100 \mathrm{~nm}$ residing in the PBG.
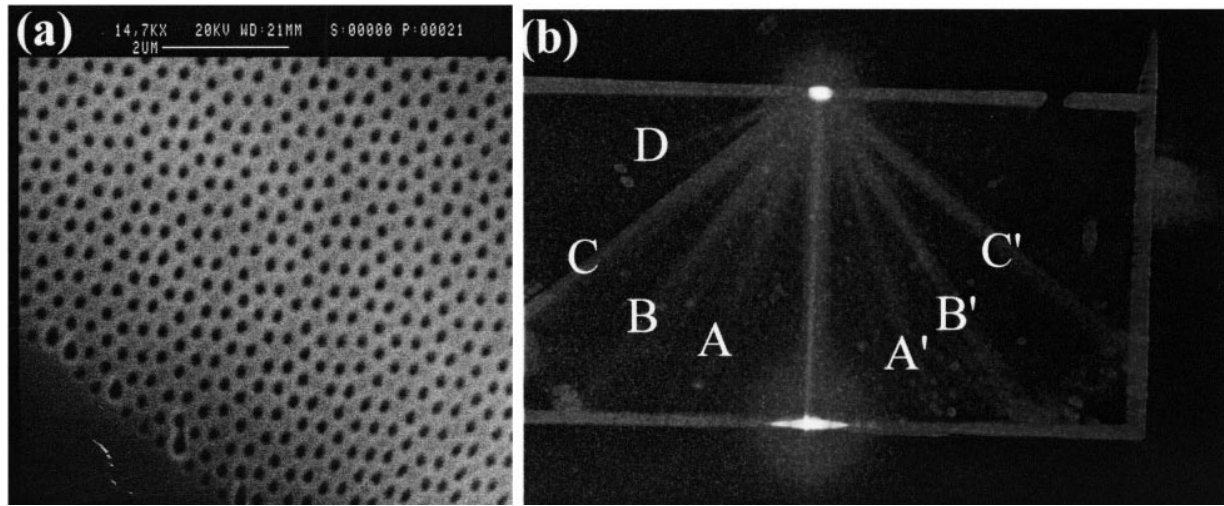

Fig. 6. SEM micrograph of fabricated photonic quascirystal (b) Photograph to demonstrate far-field diffraction at the photonic quasicrystal surface at $633 \mathrm{~nm}$. The photonic quasicrystal has a pitch of $260 \mathrm{~nm}$ and $a$ a filling fraction of 0.28 .

\section{Experimental results}

The photonic quasicrystal waveguides were fabricated for a range of parameters, and demonstrated good reproducibility and stability. Fig. 6 (a) shows an SEM micrograph of a typical device from which the pitch of $260 \mathrm{~nm}$ and fill fraction of $28 \%$ are measured.
Fig. 6 (b) shows a photograph of the small photonic quasicrystal in operation. The photonic quasicrystal (PQC) resides horizontally along the top of the photograph. The lower horizontal edge corresponds to a cleaved waveguide face. TE polarised light at $633 \mathrm{~nm}$ is focused onto the cleaved waveguide from the bottom of the photograph and is then incident perpendicular to 
Table 1

Experimental and theoretical angles of diffraction by photonic quasicrystal for $633 \mathrm{~nm}$

\begin{tabular}{llll}
\hline $\begin{array}{l}\text { Experimental } \\
\text { beam }\end{array}$ & Angle Fig. 7 & $\begin{array}{l}\text { Theoretical } \\
\text { beam }\end{array}$ & $\begin{array}{l}\text { Angle Fig. 2 } \\
\text { (a) }\end{array}$ \\
\hline A, A' & $20.9,18.8$ & 1 & 18.0 \\
B, B & $36.3,33.0$ & 2 & 34.6 \\
C, C' & $51.3,50.1$ & 3 & 51.3 \\
D & 66.3 & 4 & 67.9 \\
\hline
\end{tabular}

the PQC structure. This demonstrates the experimental far field diffraction of the quasicrystal. The angles of the diffracted beams have been compared directly with the theoretical ones shown in Fig. 2 (b). Table 1 shows good agreement between theoretical and experimental angles of diffraction. This agreement demonstrates the 12-fold symmetry of the photonic quasicrystal and confirms its successful fabrication and diffractive properties.

Transmission measurements were carried out by spectrally resolving the light propagating through the lattice in different directions. The spectra reported in Fig. 7 show the first experimental evidence of a complete secondary PBG in a quasicrystal for both TE and TM polarisations. The experiments used a white light continuum produced by focussing $1 \mu \mathrm{J} 100 \mathrm{fs}$ pulses from a regenerative amplifier tuned to $850 \mathrm{~nm}$ in $1 \mathrm{~mm}$ of sapphire. The availability of such high-brightness ultra-broadband laser sources facilitates high-accuracy transmittance measurements from 450 to $1100 \mathrm{~nm}$ through the waveguide. The use of achromatic optics and a carefully designed optical fiber spatial filter provided excellent collimation and pointing properties for coupling into the planar waveguide. Due to the scattering losses caused by the air holes, the spectra in Fig. 7(a) and (b) have been normalised to approximately account for the decreased throughput at shorter wavelengths.

Fig. 7 (a) and (b) demonstrates the highly isotropic first PBG for different angles of incident TE and TM modes respectively. Three different incident light angles are considered for each polarisation 0,6 and $12^{\circ}$. The angle of light coupled into the photonic quasicrystal structure is measured with respect to the normal along the quasicrystal surface. Fig. 7 (c) shows the experimental transmission plot for the same photonic quasicrystal with incident light along the $\Gamma \mathrm{J}$ direction. Three sharp bandgaps are clearly visible similar to predicted simulations. Additionally, it is noted that the transmission plots have a strongly modulated structure, these are dramatically different to those associated with periodic photonic crystals. This is due to the short-range disorder creating irregularities in the fine structure of the transmission plots. The extinction ratio is greater than $95 \%$ for all the frequencies in the bandgaps, with a normalised width $\Delta \omega / \omega_{0}$ ranging from $10-15 \%$.
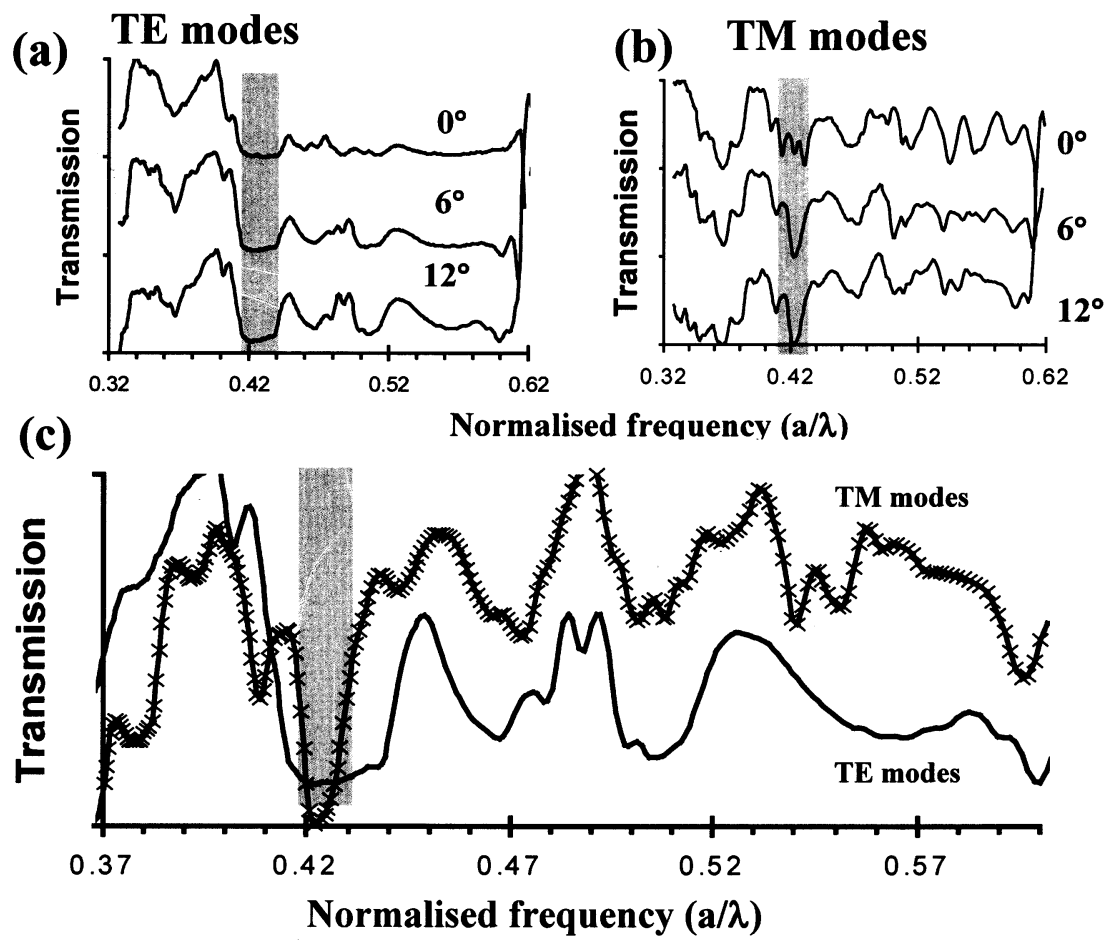

Fig. 7. Experimental transmission spectra for different angles of incident light for (a) TE modes and (b) TM modes. (c) Transmission plot for both TE and TM polarisations along the normal $(\Gamma \mathrm{J})$ incidence. The existence of the complete PBG for all polarisations is highlighted by the grey areas. 
The spectra show the same fine structure as that calculated in Fig. 4 and in particular the predicted angular and polarisation dependence. However, there is a slight discrepancy in the absolute position of the gaps. The PBG positions have been shifted by a factor of 1.03 and their widths reduced by a factor of 1.30 compared to theory. The FDTD calculations were performed using a two-dimensional (2D) model that neglects the real three-dimensional (3D) character of waveguiding in a planar structure. The in-plane $2 \mathrm{D}$ component of the wave vector $\boldsymbol{k}_{/ /}$is related to the $3 \mathrm{D}$ propagation wavevector $\boldsymbol{k}$ in the waveguide by a nonlinear relation, which results in a frequency shift of all the gaps. 3D modelling using the plane wave method for regular periodic photonic crystals shows similar behaviour [11].

\section{Conclusions}

A 12-fold symmetric photonic quasicrystal was designed and fabricated within a silicon nitride waveguide structure with a lattice pitch of $260 \mathrm{~nm}$ and $\beta=28 \%$. The properties of a photonic quasicrystal were analysed using an FDTD approach. The structure was theoretically predicted to possess a secondary complete and absolute photonic bandgap residing at a central normalised frequency of $0.412 a / \lambda$, with a gap-midgap ratio of $3.7 \%$. Additionally, this structure has the largest reported primary CAPBG to date for a low refractive index material, silicon nitride $(\varepsilon=4.08)$.

The far-field diffraction properties were predicted theroetically using the Ewald construction scheme. The experimental far-field diffraction patterns obtained were in good agreement with the theoretical predicted ones. This emphasised the 12-fold symmetric properties of the quasicrystal. Experimental transmission measurements were also performed demonstrating the experimentally proven CAPBG in a low index material. The existence of multiple CAPBGs provides prospective applications in the inhibition of spontaneous emission for active optical devices. The use of low refractive index materials and small air filling fractions helps to minimise coupling losses and scattering, improving efficiency.

The new class of photonic quasicrystals promises extremely important implications for the development of novel PBG devices directly compatible with current optical fibre devices.

\section{References}

[1] E. Yablonovitch, Phy. Rev. Lett. 58 (1987) 2059.

[2] P.R. Villeneuve, S.H. Fan, J.D. Joannopoulos, et al., Appl. Phys. Lett. 67 (2) (1995) 167.

[3] M.D.B. Charlton, M.E. Zoorob, G.J. Parker, Mat. Sci. Eng. B, 74 (2000) $17-21$.

[4] A. Barra, D. Cassagne, C. Jouanin, App. Phy. Lett. 72 (6) (1998) 627.

[5] M. Plihal, A.A. Maraduddin, Phy. Rev. B44 (1991) 8565.

[6] D. Cassagne, C. Jouanin, D. Bertho, Phys. Rev. B53 (11) (1996) 7134.

[7] C.M. Anderson, K.P. Giapis, Symmetry reduction in group 4 mm photonic crystals, Phys. Rev. B56 (1997) 7313.

[8] M.E. Zoorob, M.D.B. Charlton, G.J. Parker, Proc. of Inst. of Phys. PREP 99, 160 (1999), 5-7 January 1999 Manchester, UK.

[9] K.S. Yee, IEEE Trans. Antennas Propag. AP-14 (3) (1966) 302.

[10] M. Oxborrow, C. Henley, Phys. Rev. B48 (10) (1993) 6966.

[11] M.D.B. Charlton, G.J. Parker, Mat. Sci. Eng. B49 (1997) 155. 